\title{
Inhibición conductual, memoria de trabajo y planificación en adolescentes entre 12 y 14 años
}

\author{
Behavioral inhibition, working memory \\ and planning in adolescents between 12 and 14 years
}

Orozco Zevada María Elena morozcoz@hotmail.com

Téllez Alanís Bernarda

tellezberna@gmail.com

Centro de Investigación Transdisciplinar en Psicología, Universidad Autónoma del Estado de Morelos DOI: 10.32654/CONCIENCIAEPG.3-1.6

Para referenciar este artículo:

Orozco Zevada, M., \& Téllez, B. (2018). Inhibición conductual, memoria de trabajo y planificación en adolescentes entre 12 y 14 años. REVISTA ConCiencia EPG, 3(1), 83-99. doi: https://doi.org/10.32654/ CONCIENCIAEPG.3-1.6
Fecha de recepción: 06-05- 2018 | Fecha de aceptación: 29 -06 -2018

\section{Resumen}

71 objetivo del presente estudio fue comparar la $\checkmark$ ejecución de adolescentes entre los 12 y los 14 años en tres tareas asociadas a funciones ejecutivas: inhibición conductual, memoria de trabajo y planificación.

Se trabajó con tres grupos $(12,13$ y 14 años) cada uno con 62 participantes. Se empleó la tarea Stop signal para la inhibición conductual, para la memoria de trabajo la tarea N-Back $(1,2)$ y para la planeación la Torre de Londres modificada.

Se encontró mayor número de aciertos a los 14 años que a los 12 años en la tarea de inhibición y en la de memoria de trabajo, mientras que en la planificación no hubo diferencias. Estos hallazgos deben considerarse al realizar investigaciones del desarrollo para no agrupar esas edades sólo por un criterio aritmético.

Adicionalmente, estos resultados proporcionan información para el diagnóstico e intervención en casos de retrasos en el desarrollo de las funciones evaluadas.

Palabras clave: Inhibición conductual, memoria de trabajo, planeación, adolescencia 


\section{Summary}

7 he purpose of this study was to compare the 12 to 14 years old adolescents performance in three tasks of executive functioning: behavioral inhibition, working memory and planning. Participated three groups $(12,13$ and 14 years old) each one with 62 adolescents.

Stopsignal task was utilized to evaluate inhibition, 1-Back and 2-Back tasks to evaluate working memory, and modified Tower of London for planning.

In behavioral inhibition and working memory tasks we found a greater number of correct answers to 14 years than at 12 years, while in planning task there were no differences.

These findings should be considered when carrying out development research so as not to group these ages only by an arithmetical criterion. Additionally, these results provide information on the diagnosis and intervention in delays in the development of the evaluated functions.

Key words: Behavioral inhibition, working memory, planning, adolescence

\section{Introducción}

$\mathrm{E}$ 1 término funciones ejecutivas (FE) se le atribuye a Lezak (1982) quien afirma que son capacidades para formular y alcanzar metas mediante la planeación y acciones efectivas.

Su estudio durante la infancia y adolescencia ha despertado gran interés en diferentes ramas de la psicología debido a que se desarrollan de manera diferenciada (Anderson, 2001; Huizinga, Dolan \& Van der Molen, 2006; Van Leijenhorst,
Westenberg \& Crone, 2008; Lensing \& Elsner, 2018).

En razón de que la corteza prefrontal es la última en finalizar su desarrollo, se había considerado que las FE no estaban presentes en la infancia, sin embargo, algunas FE pueden observarse desde los primeros años de vida como es el caso de la inhibición conductual y el control atencional (Zelazo, Muller, Frye \& Marcovitch, 2003). La propuesta de Anderson, Anderson, Northam, Jacobs y Catroppa (2001) sostiene que hay funciones ejecutivas que se desarrollan en etapas más tempranas y más rápidamente que otras, lo cual ha sido corroborado por distintos autores (Huizinga, Dolan \& Van der Molen, 2006; Van Leijenhorst, Westenberg \& Crone, 2008).

Por ejemplo, se ha encontrado que en la infancia las FE presentan un desarrollo acelerado el cual disminuye su velocidad durante la adolescencia (Anderson, 2002; Best \& Miller, 2010; Diamond, Kirkham \& Amso, 2002). A pesar de que actualmente han aumentado las investigaciones sobre el desarrollo de las FE, de acuerdo a Best y Miller (2010), la mayoría de ellas se enfoca en momentos de la edad preescolar y la infancia temprana.

Existen estudios que se han enfocado en etapas tempranas del desarrollo, en especial en los primeros tres años de vida; otros se han enfocado a la etapa preescolar, entre los 3 y los 5 años (Carlson, 2005; Garon, Bryson, \& Smith, 2008; Isquith, Gioia \& Espy, 2004) mientras que otros se han dirigido a la etapa escolar que abarca de los 6 a los 11 años (Best \& Miller, 2010; Lensing \& Elsner, 2018).

El interés por las etapas tempranas influyó 
en la realización de una gran cantidad de estudios en esas edades, al igual que con los adultos jóvenes, mientras que existe menos información sobre el estado de las FE en el final de la infancia y el inicio de la adolescencia, especialmente entre los 12 y los 14 años.

Por tanto, aun se require la realización de investigaciones para conocer los cambios específicos que ocurren en las FE durante dicha etapa (Best \& Miller, 2010; Anderson et al., 2001).

En la adolescencia los sistemas cerebrales que regulan la inhibición presentan una mayor estabilidad y una integración más eficiente para demorar, detener o suprimir respuestas en curso lo cual puede observarse después de los 15 años de edad (Anderson et al., 2001; Luna, Garver, Urban, Lazar, \& Sweeney, 2004).

Existe poca información de lo que ocurre con la inhibición entre los 12 y los 14 años. Se podría pensar que se mantiene estable después de los 11 años pero el hecho de que se afirme que la mayor estabilidad se alcanza a los 15 años o inclusive hasta la edad adulta (Carriedo, Corral, Montoro, Herrero \& Rucián, 2016) es un indicador de que en los años intermedios podrían observarse diferencias.

Entre las pruebas más utilizadas para medir la inhibición de respuestas en el laboratorio se encuentran los paradigmas GoNo go y el Stop signal. La tarea Go-No go se ha empleado ampliamente para estudiar diferentes patologías psiquiátricas en las cuales se reportan fallas en dicho proceso
(Wright, Lipszyc, Dupuis, Thayapararajah \& Schachar, 2014).

Mientras que la tarea Stop signal también ha demostrado sensibilidad para dar información sobre alteraciones inhibitorias en diferentes cuadros psiquiátricos (Verbruggen \& Logan, 2008).

Estos paradigmas se sustentan en el modelo de "carreras de caballos" el cual establece una competencia entre proporcionar o detener una respuesta.

Una ejecución exitosa en estos paradigmas involucra el monitoreo para ajustar las respuestas de tal forma que exista un balance entre la consigna de responder lo más rápido posible, en deterner la respuesta o si ya se ha activado la misma detenerla si aparece la señal de alto. Contreras-González, Téllez-Alanís, Haro, Jiménez-Correa y Poblano (2015) utilizaron una tarea Stop signal con ensayos Go (oprimir un botón), con ensayos No go (no dar respuesta) y ensayos Stop (cuando aparece un estímulo Go y posteriormente se presenta la palabra ALTO se debe evitar presionar un botón).

En esta tarea, para resolver los ensayos Go se requiere de control atencional (Norman \& Shallice, 1986), para los ensayos No go se requiere de la inhibición simple (Logan, Cowan, \& Davis, 1984), mientras que para los ensayos Stop, el participante debe detener una respuesta en curso lo cual representa una inhibición compleja (Bedard et al., 2002).

La ventaja en el empleo de este paradigma es que permite reportar dos tipos de inhibición conductual. El primer tipo consiste en suprimir una respuesta ante una condición previamente especificada por lo que se trata de una inhibición 
simple, mientras que el segundo tipo, complejo, consiste en detener una respuesta en marcha ante una condición determinada.

Otra de las funciones más estudiadas en el desarrollo de las FE es la memoria de trabajo, la cual involucra mantener información en mente y manipularla de manera activa (Baddeley, 1998; Baddeley \& Hitch, 1974; Jonides \& Smith, 1999).

Esta memoria es necesaria para que el discurso, la lectura y la escritura tengan sentido. Existen estudios que la han evaluado a partir de los dos años de edad (Miller, 2015; Zelazzo, Muller, Frye \& Marcovitch, 2003).

A pesar de su emergencia temprana, se ha observado que alcanza su desarrollo pleno entre los 16 y los 20 años de edad (Luna et al., 2004; Luciana, Conklin \& Yarger, 2005), aunque para algunos su desarrollo se estabiliza a los 15 años (Carriedo et al., 2016).

En un estudio realizado con diferentes tareas de memoria de trabajo (visual, espacial, fonológico) se encontró que en participantes de 4 a 15 años, agrupados en bloques de dos o tres años, había incrementos lineales hasta la adolescencia (Gathercole, Pickering \& Wearing, 2004). Sin embargo, no es claro si hay diferencias significativas entre cada año o si hay etapas conformadas por varios años.

Ahora bien, el paradigma N-Back ha sido ampliamente utilizado para estudiar las bases neurológicas de los procesos de memoria de trabajo (Owen, McMillan, Laird \& Bullmore, 2005; Redick \& Lindsey, 2013; Contreras et al., 2015) ya sea verbal o auditiva.

Esta tarea consiste en presentar en una pantalla estímulos repetidos con intervalos de 1, 2 ó 3 interferencias (1-Back, 2-Back, 3-Back) y para resolverla se requiere de la actualización y manipulación de la memoria, así como de ignorar los estímulos irrelevantes.

En el presente estudio se decidió utilizar una tarea donde los estímulos fueran formas sin sentido, con el objetivo de evitar la ayuda de lo semántico para recordar letras o números presentados en la pantalla, el cual ya ha sido utilizado por Contreras et al. (2015).

Con respecto a la validez de la tarea N-Back, es importante señalar que ésta ha mostrado relación con tareas de span de dígitos pero no con tareas de span de lectura (Jaeggi, Buschkuehl, Perrig, \& Meier, 2010; Redick \& Lindsey, 2013) todas ellas consideradas como medidas tradicionales de la memoria de trabajo.

Por otro lado, una función ejecutiva que se desarrolla más tardíamente es la planeación (Van den Heuvel, Groenwegen, Barkof, Lazeron, Van Dyck \& Veltman, 2003). Ésta consiste en determinar una meta y los pasos necesarios para alcanzarla, así como la secuencia de los mismos.

Para ello se requiere de una representación mental de la situación actual y de la meta que se quiere alcanzar, lo cual en muchas ocasiones no se distingue fácilmente. Anderson et al. (2001) han descrito tres aspectos escenciales para resolver un problema. En primer lugar el comportamiento organizado claramente dirigido a conseguir la meta.

En segundo lugar las metas intermedias que se tienen que alcanzar y en tercer lugar las acciones que llevan a la meta. 
La capacidad de planeación aumenta con la edad y alcanza su desarrollo pleno entre los 20 años, edad en la que tienen lugar los retos más complejos y demandantes (Anderson, Jacobs \& Anderson, 2008). Para Diamond y Lee (2011), se puede hablar de planeación efectiva después de los 11 años de edad y coincide en que alcanza su máximo desarrollo después de los 20 años.

Nuevamente nos encontramos con la incognita de lo que sucede en la adolescencia temprana respecto al desarrollo de esta función que ha sido considerada fundamental para alcanzar metas y establecer los pasos para resolver problemas tanto en el ámbito escolar como laboral, además de la vida cotidiana.

Además, la Torre de Londres se ha utilizado con frecuencia para evaluar la habilidad para planear (Sullivan, Riccio \& Castillo, 2009). Inicialmente fue desarrollada por Shallice (1982) para medir la capacidad de planeación y resolución de problemas en pacientes con daño frontal.

Se consideran tres indicadores de planeación: el tiempo inicial o tiempo de planeación, el número de movimientos excedentes y el número de soluciones perfectas.

Mayor tiempo inicial y mayor número de soluciones perfectas, aunado a un menor número de movimientos excedentes, son indicadores de una buena planeación.

La Torre de Londres empleada por Shallice (1982) cuenta con 10 ensayos y fue diseñada para identificar deterioro en los procesos de planeación en pacientes, mientras que la modificación aquí empleada, propuesta por Ocampo y Téllez (2015), consta de 21 ensayos y fue pensada para aplicarse a participantes sanos por lo que presenta un mayor grado de dificultad.

La Torre de Londres ha desmostrado tener buenas propiedades psicométricas, especificamente la confiabilidad evaluada con el método de las dos mitades y la consistencia interna (Kaller, Unterrainer \& Stahl, 2012) así como también la validez de constructo (Debelak, Egle, Köstering \& Kaller, 2016).

La información sobre el desarrollo de las FE antes mencionadas, entre los 12 y 14 años, es poco clara. Por ejemplo, existen estudios que dicen que la inhibición presenta un desarrollo importante entre $\operatorname{los} 8$ y los 14 años (Thibaut, French \& Vezneva, 2010), pero no se conoce que sucede en ese lapso de 6 años, lo cual es un largo período en la vida de una persona.

Algo similar sucede con la memoria de trabajo, los rangos de edad que algunos autores han estudiado de manera general van de los 7 a los 13 años (Diamond, Kirkham \& Amso, 2002), nuevamente sin hacer distinciones en etapas más cortas.

En cuanto a la planeación existen investigaciones que indican que el desempeño máximo se alcanza hacia los 15 años de edad (Welsh, Pennington \& Groisser, 1991) o hasta después de los 20 años (Anderson et al., 2008; Diamond \& Lee, 2011), nuevamente sin entrar en detalles sobre los tres años que se han comparado en el presente trabajo. Los estudios sobre el desarrollo de las FE no especifican si existen diferencias en el desarrollo de las mismas entre los 12 y 14 años, pareciera que en esa etapa no hay diferencias y que a los 15 años se diera un mayor 
desarrollo.

La hipótesis del presente trabajo es que existen diferencias en el desarrollo entre los 12 , 13 y 14 años. El objetivo del estudio consistió en determinar el nivel de desarrollo de la inhibición de respuestas, la memoria de trabajo y la planeación al inicio de la adolescencia, entre los 12, 13 y 14 años de edad.

Laimportancia de establecer diferencias entre estas edades radica en que en esta etapa ocurren cambios en la vida de los adolescentes. Uno de los más significativos es el ingreso a la educación secundaria, que es un reto importante porque el adolescente debe enfrentarse a situaciones nuevas que exigen un esfuerzo cognitivo mayor que en la primaria.

El estudiante de secundaria necesita organizar su tiempo y sus actividades para adaptarlas a siete $\mathrm{u}$ ocho materias diferentes por día con diferentes maestros, lo que no ocurría en la escuela primaria.

Para un buen desempeño académico necesita hacer frente a situaciones novedosas que requieren FE complejas como la planeación. Con el conocimiento del estado que deben tener las funciones ejecutivas evaluadas en el presente estudio será posible diseñar programas de intervención educativa que permita mejorar las FE de los adolescentes que lo requieran para aumentar el desempeño académico.

Adicionalmente se podrá dar seguimiento a estos adolescentes para apoyarlos en su trayectoria académica que pudo verse afectado por un problema derivado del funcionamiento ejecutivo.

\section{Método}

Diseño: La presente investigación tiene un diseño cuasi experimental con grupo de comparación no equivalente. Según Hernández Sampieri, Fernández Collado y Baptista Lucio (2006), las investigaciones cuasi experimentales consisten en estudios que manipulan al menos una variable independiente para observar su relación con otras variables.

Difieren de las experimentales en que los grupos están intactos y por lo tanto pueden resultar no equivalentes.

Participantes: $\quad$ Participaron 186 adolescentes, 94 hombres y 92 mujeres, con edades comprendidas entre los 12 y los 14 años de edad, estudiantes regulares de una escuela secundaria ubicada al poniente de la capital de una entidad federativa en México, la cual presenta un grado de marginación muy bajo de acuerdo a los datos de la Secretaría de Educación Pública (SNIEE, 2014). Se utilizó un muestreo no probabilístico por convenencia.

En la tabla 1 se muestran la distribución de los participantes por sexo y edad. 
Tabla 1

Distribución por grupo de edad y género

de los participantes

\begin{tabular}{llll}
\hline Edad (años) & Femenino & Masculino & Total \\
\hline 12 & 27 & 35 & 62 \\
13 & 37 & 25 & 62 \\
14 & 28 & 34 & 62 \\
Total & 92 & 94 & 186 \\
\hline
\end{tabular}

Instrumentos: Inhibición conductual. Se utilizó el paradigma Stop signal.

Al inicio se presentaron ocho ensayos de práctica.

Durante la tareas se presentaron 200 estímulos tipo Go en los que hay que presionar una tecla ante la presencia de un estímulo (círculo azul) ubicado en siete de ocho posibles ubicaciones en una matriz de 3 x 3 (la posición del centro estuvo deshabilitada); 30 estímulos tipo No go en los que no hay presionar una tecla ante la presencia del estímulo en la esquina superior izquierda de la matriz; y 20 estímulos Stop en los que, después de aparecer el estímulo Go, adicionalmente aparecía la palabra ALTO y se debía suspender la respuesta ya activada, la latencia de esta señal de detención varió entre 150,200 y $250 \mathrm{mseg}$.

Al inicio del ensayo se presentó un asterisco como punto de fijación (1000 mseg), el estímulo duraba 1000 milisegundos y se contaba con un tiempo máximo de 2500 mseg para recibir la respuesta a partir de la presentación del estímulo.
La duración total de la tarea fue de 15 minutos. Para la calificación de esta prueba se consideraron los aciertos de los estímulos No go y los estímulos Stop.

Memoria de trabajo. Se utilizó la tarea N-Back con 80 estímulos para cada condición, los cuales fueron figuras abstractas sin significado, 57 no se repitieron y 23 repetidos (ensayos N-back) con interferencia de 1 o 2 estímulos, según fuera el caso.

Al inicio se presentaron 16 ensayos de práctica para garantizar la comprensión de la tarea. Los estímulos se presentaron durante 1200 mseg con un tiempo máximo para dar la respuesta de $2500 \mathrm{mseg}$ a partir del inicio de la presentación del estímulo. $\mathrm{P}$

ara la calificación de la prueba se consideraron los aciertos al oprimir una tecla cuando el participante reconoció el estímulo visto en 1 o 2 estímulos anteriores. Cada tarea N-back duraba alrededor de 4 minutos.

Torre de Londres modificada a 21 ensayos. La tarea implica la planeación y organización de la tarea, el monitoreo de la propia actividad y la capacidad 
para cambiar de estrategia en caso necesario. Se emplean dos tableros con tres barras de diferente tamaño y tres esferas de diferente color, con ella se presenta una configuración en el tablero del evaluador, la cual debe ser reproducida en el menor número de movimientos posibles por parte del evaluado, además se deben de respetar dos reglas.

Se tomaron en cuenta tres indicadores: El número de movimientos excedentes a lo establecido para cada ejercicio, el número de soluciones con el mínimo de movimientos o perfectas y la suma del tiempo de planeación de todos los ensyos. Esta prueba tenía una duración aproximada de 25 minutos.

Procedimiento: Para llevar a cabo el estudio se solicitaron las autorizaciones pertinentes con las autoridades de la Secretaría de Educación Pública y con las autoridades del plantel educativo.

Asimismo se llevaron a cabo reuniones con los maestros y padres de familia para informarles sobre el estudio y se obtuvo el consentimiento informado de los padres y el asentimiento informado de los participantes, como lo indican los principios de ética de la Comisión Nacional de Bioética (Lolas, Quezada \& Rodríguez, 2006).

Los datos se recolectaron entre noviembre de 2014 y marzo de 2015. La aplicación de los instrumentos se llevó a cabo en la biblioteca de la secundaria donde se instalaron 5 computadoras personales a las que previamente se instaló el software de evaluación. La aplicación se llevó a cabo en dos sesiones por participante.
En una sesión se aplicaron las tareas computarizadas (Stop signal y N-Back) y en otra sesión se aplicó la Torre de Londres modificada. La aplicación de las pruebas fue contrabalanceada.

Para evaluar los supuestos requeridos se aplicó la prueba de normalidad KolmogorovSmirnov, para la homogeneidad de las varianzas entregrupos la prueba de Levene y para la esfericidad intragrupo la prueba de Mauchly (sólo en las tareas de inhibición y memoria de trabajo). Para comparar los aciertos de las tareas Stop signal y N-Back se realizó un Análisis de Varianza (ANOVA) mixto con el factor entre-grupos edad (12, 13 y 14 años) en ambos análisis.

En Stop signal el factor intra-grupos fue Tipo de inhibición (No go x Stop) y en N-Back fue carga de memoria de trabajo (1Back x 2-Back). En el caso de la Torre de Londres se utilizaron ANOVA univariados para comparar el tiempo de planeación, las soluciones perfectas y los movimientos excedentes entre los tres grupos de edad.

Cuando se requirió (en las interacciones $\mathrm{y}$ en el factor grupo, significativos) se realizaron análisis pos hoc con la prueba HSD de Tukey y en los casos que no se cumplió con la homogeneidad de las varianzas entre grupos se calculó la prueba Welch-James para comparar medias con varianzas desiguales.

Para todas las pruebas aplicadas el nivel de significancia requerido fue $\leq .05$ 


\section{Resultados}

Con respecto a los supuestos, de las siete variables evaluadas (2 inhibición, 2 de memoria de trabajo y 3 de planeación) la mayoría mostraron que no cumplían con el supuesto de normalidad, a excepción de los movimientos excedentes en la Torre de Londres.

Sin embargo, se decidió continuar con el análisis de varianza pues éste es robusto ante las violaciones de normalidad (Balluerka \& Vegara, 2002; Schmider, Ziegler, Danay, Beyer \& Bühner, 2010).

Con respecto a la homogeneidad de las varianzas entre grupos sólo en la tarea de inhibición se violó este supuesto $(\mathrm{p}<.01$ en No go y Stop) por lo que se aplicó la prueba Welch-James ( $\mathrm{F}$ con distribución asintótica -Fa-).

Mientras que, en las tareas con diseño mixto, inhibición y memoria de trabajo, no se encontraron violaciones a la esfericidad con la prueba de Mauchly.

\section{Inhibición}

Con respecto a la edad se encontraron diferencias significativas $(\mathrm{Fa} 2,121=10.4$, $\mathrm{p}<.001)$ el análisis poshoc de Tukey indicó que las diferencias están entre el grupo de 12 años $(66 \% \pm 1.7)$ y el de 14 años $(76 \% \pm 1.7)$.

La media del grupo de 13 años fue de $(71 \% \pm 1)$, pero no se diferenció significativamente de los otros dos grupos. También hubo diferencias de acuerdo al tipo de inhibición $(\mathrm{F} 1,183=96, \mathrm{p}<=.001)$ puesto que en los ensayos No go $(79 \% \pm 1.5$; media \pm error estándar) la ejecución fue mayor que ante los Stop $(63 \% \pm 1.1)$. En la interacción edad por tipo de inhibición $(\mathrm{F} 2,183=15, \mathrm{p}<.001)$ hubo diferencias confirmadas por el análisis pos hoc de Tukey, el cual reveló que en los ensayos No go el grupo de 14 años tuvo mayor ejecución que los otros dos grupos, y que en los ensayos Stop el grupo de 12 años tuvo menor ejecución que los de 13 y 14 años (Figura 1).

\section{Memoria de trabajo}

El factor carga $(\mathrm{F} 1,183=265, \mathrm{p}<.001)$ fue significativamente diferente al igual que el factor edad $(F 2,183=3.4, \quad p=.034)$, mientras que la interacción carga por edad no resultó significativa.

El porcentaje de aciertos fue mayor en la tarea 1 -Back (84.4\% $\% 1.2)$ que en la 2 -Back $(52.6 \% \pm 1.5)$ y el análisis pos hoc mostró diferencias entre los $12(64.9 \% \pm 1.7)$ y $14(70.5 \% \pm 1.7)$ años de edad. El grupo de 13 años mostró una media de $70.5 \% \pm 1.7$ pero no fue diferente al grupo de 12 años.

\section{Planeación}

En la tabla 1 se muestran las medias y los errores estadar de los movimientos excedentes, la suma del tiempo de planeación y el número de las soluciones. No se encontraron diferencias de acuerdo a la edad en ninguno de las variables evaluadas. 
Tabla1

Media (error estandar) de los tres indicadores de la Torre de Londres modificada

\begin{tabular}{llll}
\hline Grupo de edad & Movimientos excedentes & Soluciones perfectas & $\begin{array}{l}\text { Tiempo de } \\
\text { planeación en } \\
\text { segundos }\end{array}$ \\
\hline 12 años & $62 \pm 2$ & $8 \pm .3$ & $75 \pm 7$ \\
13 años & $61 \pm 2$ & $9 \pm .3$ & $67 \pm 7$ \\
14 años & $57 \pm 2$ & $9 \pm .3$ & $85 \pm 7$ \\
\hline
\end{tabular}

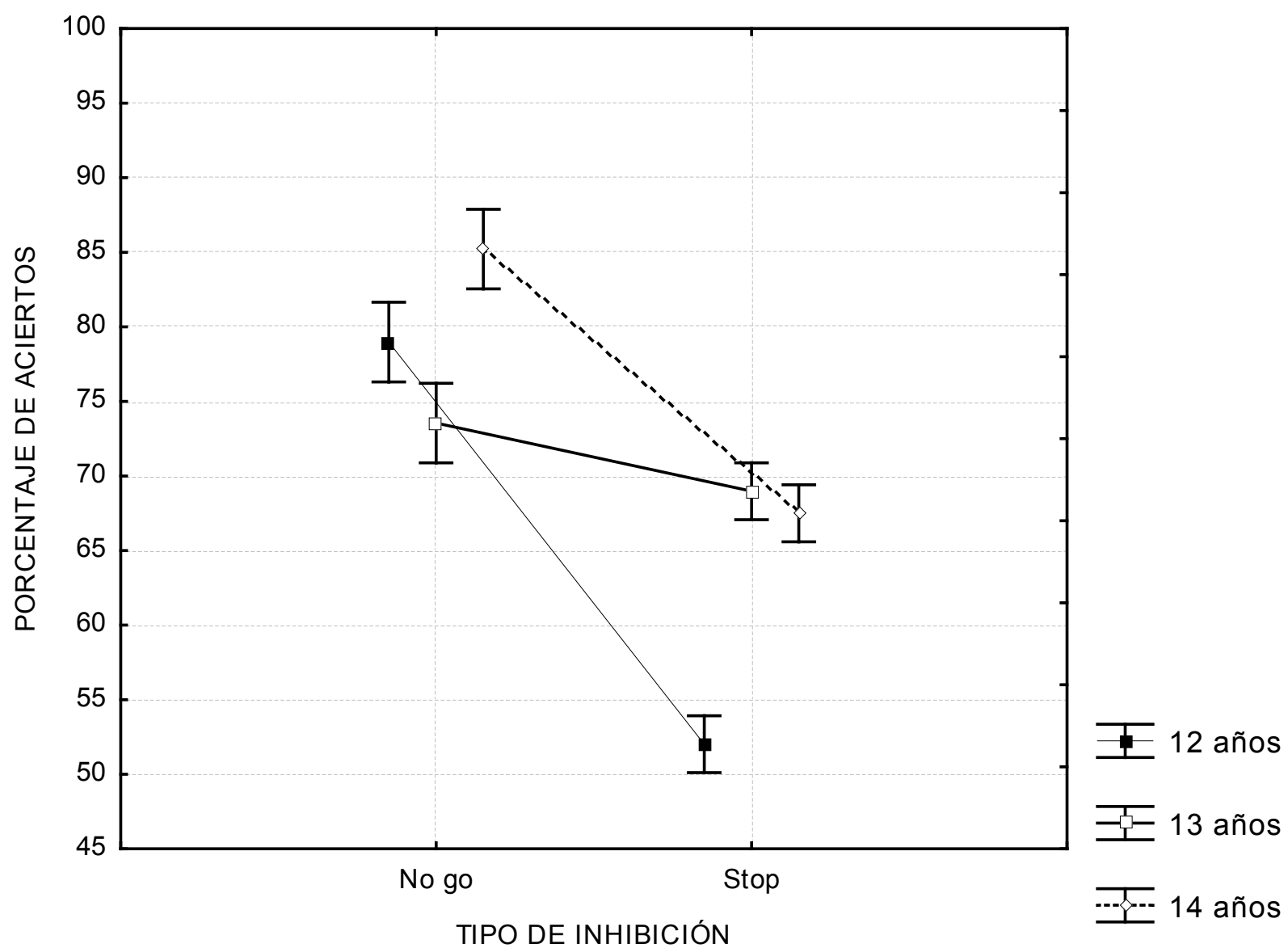

Figura 1. Porcentaje de aciertos en la tarea Stop signal ante los ensayos No go y Stop de acuerdo al grupo de edad 


\section{Discusión}

El objetivo del presente estudio fue comparar la ejecución de adolescentes entre $\operatorname{los} 12, \operatorname{los} 13$ y los 14 años de edad en tres tareas asociadas a funciones ejecutivas: inhibición conductual, memoria de trabajo y planificación.

Los resultados del presente estudio muestran que el desarrollo de la inhibición, medida con la prueba Stop signal, se encuentra en etapas distintas entre los 12 y los 14 años. Según Anderson et al. (2001), la inhibición se mantiene estable entre los 11 y los 15 años, cuando alcanza su máximo desarrollo.

Sin embargo, en el presente estudio encontramos un diferencia entre el tipo de inhibición puesto que ante el estímulo No go la ejecución se encuentra en un nivel similar entre los 12 y 13 años y su desempeño aumenta a los 14 años.

Estos resultados son similares a los obtenidos por Hooper (2004) y por Luciana, Collins, Olson y Schissel (2009) quienes reportaron un desarrollo de la inhibición evaluada con el paradigma No go, reflejado por la disminución de falsas alarmas. Sin embargo ellos compararon grupos de edad que incluyen dos o tres años: entre 9-10 años y los 14-17 años (Hooper, 2004), y 9-11, 12-14 y 15-17 años (Luciana et al., 2009); mientras que en el presente estudio encontramos diferencias entre grupos de edad más cercanos.
Ahora bien, la detención de una respuesta ya iniciada, la cual es más compleja en tanto que tiene menor porcentaje de aciertos que la inhibición ante un estímulo específico, se diferencia a partir de los 13 años, mientras que a los 12 años presenta menor ejecución.

Este resultado de la interacción llama la atención puesto que se esperaría que la diferencia se diera también a los 14 años, en tanto que se trata de una proceso más complejo. Se deben realizar estudios adicionales para confirmar y explorar este resultado.

Adicionalmente, al comparar estos resultados con los obtenidos por Contreras et al. (2015) podemos observar que a los participantes de 14 años aun les falta alcanzar los niveles de ejecución del grupo de adultos de esa muestra (No go $97.3 \%$ y Stop $89.6 \%$ ), es decir que la inhibición conductual, tanto simple como compleja, aún se continúa desarrollando de la adolescencia a la edad adulta (Carriedo et al. 2016).

También los resultados nos indican que la ejecución es mayor en los ensayos No go que en los Stop, este patron de ejecución se ha reportado en otros estudios indicando que un tipo de ejecución es más dificil que otro (Contreras et al., 2015). Y cuando se explora esta interación vemos que en la tarea más sencilla (No go) el grupo de 14 años tiene la ventaja, mientras que en la más compleja (Stop) el grupo de 12 años tiene menor ejecución que los otros dos grupos.

Esta interacción es interesante en tanto 
debemos considerar el tipo de tarea con el que es evaluada la inhibición conductual para señalar si ésta madura o no en un grupo específico de edad.

Con respecto a la memoria de trabajo se encontró que a mayor carga la ejecución disminuye en los tres grupos de edad sin diferencias entre ellos. Es decir, la tarea 2-Back es más compleja que la 1-Back. Con respecto a la edad el grupo de 14 años es el que presenta mayor ejecución que el grupo de 12 años.

Tanto Hooper (2004) como Luciana et al. (2009) reportan mejoras en la memoria de trabajo empleando los resultados de digitos directos e inversos en conjunto. Al igual que en las tareas de inhibición, los resultados muestran que la memoria de trabajo aún se continuará desarrollando en tanto que los participantes obtuvieron porcentajes de aciertos menores a los obtenidos por adultos en el estudio de Contreras et al. (2015) (1-Back 95\% y 2-Back 85\%).

La interacción entre ambas variables no demostró diferencias específicas en algún tipo de carga en algún grupo de edad. Esto indica que no hay algún avance de cuerdo a la edad dependiendo si se trata de 1-Back o 2-Back, es decir, el nivel de complejidad provoca diferencias en los grupos de edades.

Por otro lado, anteriormente se consideraba que la planeación se desarrollaba hasta la juventud, lo que implica que los adolescentes no contaban con la capacidad de planear. Al contrario, Diamond y Lee (2011) consideran que la capacidad de planear se encuentra presente a partir de los 12 años y que efectivamente alcanza su desarrollo al final de la adolescencia, alrededor de $\operatorname{los} 21$ años.
Con respecto a los grupos de edad evaluados, los resultados del presente estudio muestran que en la Torre de Londres no hay diferencias entre los 12 y 14 años en ninguno de los indicadores evaluados: tiempo de planeación, movimientos excedentes $\mathrm{y}$ soluciones perfectas. Es decir, se encuentran en una etapa de desarrollo similar.

Luciana et al. (2009) aplicaron la Torre de Londres digitalizada con 12 problemas de 2 a 5 movimientos y encontraron mejoras en el número de soluciones perfectas entre 9-11 años y 12-17 años.

Como puede notarse uno de los grupos evaluados incluye las tres edades comparadas en el presente estudio, es decir, se toman dicahas edades como un grupo compacto.

La planeación, al ser un proceso complejo (Diamond \& Lee, 2011) requiere tal vez de mayor tiempo de maduración para su alcanzar avances, lapsos de tres o más años, o tal vez es más sensible a diversas variables del entorno social que deben ser consideradas al evaluar dicho proceso.

Por tanto, la agrupación de estas tres edades (12, 13 y 14 años) para su investigación es adecuada, además, esto sugiere que se puede trabajar con la estimulación de este proceso dentro de la escuela secundaria organizando actividades similares para los tres grados escolares.

Entre las limitaciones del estudio podemos reconocer que un mayor número de participantes en la muestra siempre es positivo para aumentar la potencia estadística de los resultados, además de que se debiera igualar el número de hombres y mujeres para 
explorar si existen diferencias debido al sexo como ya se reportó anteriormente (Boelema et al., 2014; Lensing \& Elsner, 2018).

Asimismo, es importante considera otras variables que se relacionan positivamente con la edad en el desarrollo de las funciones ejecutivas como la velocidad de procesamiento, el estado socieconómico y la habilidad verbal (Lensing \& Elsner, 2018).

Como un acierto de la investigación se reconoce que las tareas utilizadas nos permiten comparar dos niveles de complejidad, sin embargo no se utilizaron diferentes tipos de tareas para cada uno de los procesos, lo cual disminuye la validez de contenido de las variables del estudio.

Otro acierto es que, al existir estudios previos con las pruebas utilizadas, se logró comparar los resultados con estudios realizados en adultos. Por último reconocemos que se trata de un estudio transversal con las limitaciones que ello implica.

Podemos observar que las FE evaluadas siguen un patrón distinto en estos grupos de edad, en algunos hay diferencia y en otro no. Esta información es relevante para detectar retrasos en el desarrollo de estas funciones con el objetivo de diseñar intervenciones para fortalecer a las mismas, tanto de caracter académico en el contexto escolar lo cual apoya de manera directa a la mejora del desempeño académico (Diamod \& Lee, 2011), como en entornos clínicos cuando hay problemas de conducta o familiares.

\section{Conclusión}

En conjunto, estos resultados muestran que a los 14 años hay un desarrollo mayor que a los 12 años en las tareas que miden inhibición conductual y memoria de trabajo.

Por lo que en futuros estudios sobre el desarrollo deben considerarse las diferencias entre estas edades y no realizar un agrupamiento de estos tres años de edad para evaluar la ejecución en los adolescentes.

Lo anterior no sucede en la tarea de planeación debido a que los tres grupos de edad muestran un desarrollo similar en la tarea de planeación, y por tanto estas tres edades sí pueden ser agrupadas para estudiar el desarrollo de las funciones ejecutivas.

Finalmente, cabe mencionar que el estudio de las funciones ejecutivas en etapas específicas de la adolescencia permite un mayor conocimiento sobre el desarrollo de las mismas lo cual se traduce en información de apoyo para las actividades de intervención en el ámbito académico y clínico.

Con este conocimiento los especialistas y los maestros podrán distinguir y aplicar el diseño estrategias específicas por función ejecutiva para estimular el desarrollo de quiene presenten retrasos o disfunciones. 


\section{Referencias}

Anderson, P. (2002). Assessment and development of executive function (EF) during childhood. Child neuropsychology, 8(2), 71-82. doi: 10.1076/chin.8.2.71.8724.

Anderson, V. (2001). Assesing executive functions in children: Biological, psychological, and developmental considerations. Pediatric rehabilitation, 4(3), 119-136. doi: $10.1080 / 13638490110091347$

Anderson, V., Anderson, P., Northam, E., Jacobs, R. \& Catroppa, C. (2001). Development of executive functions through late chilhood and adolescence in an Australian sample. Development neuropsychology, 20 (1), 385406. doi: 10.1207/S15326942DN2001_5.

Anderson, V., Jacobs, R. \& Anderson, P. (2008). Executive functions and the frontal lobes: A life span perspective. New York: Taylor \& Francis.

Baddeley, A. \& Hitch, G. (1974). Working memory. Oxford: Clarendon Press.

Baddeley, A. (1998). The central executive: A concept and some misconceptions. Journal of the international neuropsychological society, 4,(5) 523-526. doi: 10.1017/ S135561779800513X.

Balluerka, L. N. \& Vergara, A. I (2002). Diseños de investigación experimental en psicología: modelos y análisis de datos mediante el SPSS 10.0. Madrid: Pearson Educación.

Bedard, A., Nichols, S., Barbosa, J., Schachar, R. Logan, G. \& Tannock R. (2002). The development of selective inhibitory control across the life span. Development Neuropsychology, 21(1), 93-111. doi: 10.1207/S15326942DN2101_5.

Best, J. \& Miller, H. (2010). A developmental perspective on executive function. Child development, 81(6), 1641-1660. doi: 10.1111/j.1467-8624.2010.01499.x.

Boelema, S. R., Harakeh, Z., Ormel, J., Hartman, C. A., Vollebergh, W. A. \& van Zandvoort, M. J. (2014). Executive functioning shows differential maturation from early to late adolescence: Longitudinal findings from a TRAILS study. Neuropsychology, 28(2), 177.

Carlson, S. (2005). Developmentally sensitive measures of executive function in preschool children. Developmental Neuropsychology, 28(2), 595-616. doi:10.1207/s15326942dn2802_3.

Carriedo, N., Corral, A., Montoro, P. R., Herrero, L. \& Rucián, M. (2016). Development of the updating executive function: From 7-year-olds to young adults. Developmental psychology, 52(4), 666-678.

Contreras-González, N., Téllez-Alanís, B., Haro, R., Jiménez-Correa, U. \& Poblano, A. (2015). Executive dysfunction in patients with chronic primary insomnia treated with clonazepam. Neurological Research, 37(12), 1047-1053. doi: 10.1080/01616412.2015.1114740. 
Debelak, R., Egle, J., Köstering, L. \& Kaller, C. P. (2016). Assessment of planning ability: Psychometric analyses on the unidimensionality and construct validity of the Tower of London Task (TOL-F). Neuropsychology, 30(3), 346. doi: $10.1037 /$ neu0000238.

Diamond, A. \& Lee, K. (2011). Interventions shown to aid executive function development in children 4 to 12 years old. Science, 333(6045), 959-964. doi: 10.1126/science.1204529.

Diamond, A., Kirkham, N. \& Amso, D. (2002). Conditions under which young children can hold two rules in mind and inhibit a prepotent response. Developmental psychology, 38(3), 352-362. doi: 10.1037//0012-1649.38.3.352 .

Garon, N., Bryson, S. \& Smith, I. (2008). Executive function in preschoolers: a review using an integrative framework. Psychology Bulletin, 134(1), 1032-1053. doi: 10.1037/0033-2909.134.1.31.

Gathercole, S., Pickering, B. \& Wearing, H. (2004). The structure of working memory from 4 to 15 years of age. Developmental psychology, 40(2), 177190. doi: 10.1037/0012-1649.40.2.177.

Hernández Sampieri, R., Fernández Collado, C. \& Baptista Lucio, P. (2010). Metodología de la investigación. México: McGraw-Hill.
Hooper, C. L. (2004). Adolescents' performance on the Iowa Gambling Task: implications for the development of decision making and ventromedial prefrontal cortex. Developmental psychology, 40(6), 11481158. doi: 10.1037/0012-1649.40.6.1148.

Huizinga, M., Dolan, C. \& Van der Molen, M. (2006). Age-related change in executive function: Developmental trends and a latent variables analysis. Neuropsychologia, 44(11), 2017-2023. doi: 10.1016/j. neuropsychologia.2006.01.010.

Isquith P., Gioia G. \& Espy K. (2004). Executive function in preschool children: examination through everyday behavior. Development neuropsycholoogy, 26(1), 403-422. doi: 10.1207/s15326942dn2601_3.

Jaeggi, S. M., Buschkuehl, M., Perrig, W. J. \& Meier, B. (2010). The concurrent validity of the N-back task as a working memory measure, Memory, 18(4), 394-412. doi: $10.1080 / 09658211003702171$

Jonides, J. \& Smith, E. (1997). The architecture of working memory. En M. Rugg (Ed.), Cognitive neuroscience, pp. 243-276. Cambridge, MA: MIT Press.

Kaller, C. P., Unterrainer, J. M., \& Stahl, C. (2012). Assessing planning ability with the Tower of London task: Psychometric properties of a structurally balanced problem set. Psychological Assessment, 24(1), 46-53. doi: 10.1037/a0025174 
Lensing, N. \& Elsner, B. (2018). Development of hot and cool executive functions in middle childhood: Three-year growth curves of decision making and working memory updating. Journal of experimental child psychology, 173, 187-204. doi: 10.1016/j. jecp.2018.04.002

Lezak, M. (1982). The problem of assesing executive functions. International Journal of Psychology, 17(1-4), 281-297. doi: 10.1080/00207598208247445.

Logan, G., Cowan, W. \& Davis, K. (1984). On the ability to inhibit simple and choice reaction time responses: a model and a method. Journal of experimental psychology: Human perception and performance, 10(2), 276291. doi: 10.1037//0096-1523.10.2.276.

Lolas, F. Quezada, A. \& Rodríguez, E. . (2006). Investigación en salud. Dimensión ética. México: SSP.

Luciana, M., Collins, P., Olson, E., Schissel, A. (2009). Tower of London Performance in Healthy Adolescents: The Development of Planning Skills and Associations With Self-Reported Inattention and Impulsivity. Developmental Neuropsychology, 34(4), 461-475. doi: 10.1080/87565640902964540.

Luciana, M., Conklin, H. \& Yarger, R. (2005). The development of nonverbal WM and executive control processes in adolescents. Child development, 76(3), 697-712. doi: 10.1111/j.1467-8624.2005.00872.x.
Luna, B., Garver, K., Urban, T., Lazar, N. \& Sweeney, J. (2004). Maturation of cognitive processes from late childhood to adulthood. Child development, 75(5), 1357-1372. doi: 10.1111/j.14678624.2004.00745.x.

Miller, S. (2015). Examining Executive Function in the Second Year of Life: Coherence, Stability, and Relations to Joint Attention and Language. Developmental Psychology, 51(1), 101114. doi: $10.1037 / \mathrm{a} 0038359$.

Norman, D. \& Shallice, T. (1986). Attention to action. Willed and automatic control of behavior. En G. S. R.J. Davidson, Consciousness and Self-regulation, Vol. 4, pp. 1-19. New York: Plenum Press.

Ocampo, A. \& Téllez, B. (2015). Planeación mental y memoria de trabajo durante la resolución de la torre de Londres. E pp. (41-70). Cuernavaca: UAEM-Bonilla Artigas.

Owen, A., McMillan, K., Laird, A. \& Bullmore, E. (2005). N-Back working memory paradigm: A Meta-Analysis of Normative Functional Neuroimaging Studies. Human Brain Mapping, 25(1), 25-46. doi: 10.1002/hbm.20131.

Redick, T. S. \& Lindsey, D. R. (2013). Complex span and n-back measures of working memory: a meta-analysis. Psychonomic bulletin \& review, 20(6), 1102-1113. doi: 10.3758/s13423-0130453-9. 
Schmider, E., Ziegler, M., Danay, E., Beyer, L. \& Bühner, M. (2010). Is it really robust? Methodology, 6(4), 147-151. doi: 10.1027/1614-2241/a000016.

Shallice, T. (1982). Specific impairments of planning. London: Real Society of London.

SNIEE. (2014). Sistema Nacional de Información Estadística Educativa. Recuperado de http://www.enlace.sep. gob. $\mathrm{mx} /$ content $/ \mathrm{ba} / \mathrm{docs} / 2011 / \mathrm{base}$ de_datos_completa/17_RESUL_ESC_ GRADO ASIGNATURA.xls

Sullivan, J. R., Riccio, C. A. \& Castillo, C. L. (2009). Concurrent validity of the tower tasks as measures of executive function in adults: a meta-analysis. Applied neuropsychology, 16(1), 62-75. doi: $10.1080 / 09084280802644243$.

Thibaut, J. P., French, R. \& Vezneva, M. (2010). The development of analogy making in children: Cognitive load and executive functions. Journal of experimental child psychology, 106(1), 1-19. doi: 10.1016/j.jecp.2010.01.001.

Van den Heuvel, O., Groenwegen, H., Barkof, F., Lazeron, R. Van Dyck, R. \& Veltman, D. (2003). Frontostriatal system in planning complexity: a parametric functional magnetic resonance version of tower of London task. Neuroimage, 18(2), 367-374. doi: 10.1016/S10538119(02)00010-1.
Van Leijenhorst, L., Westenberg, P. M. \& Crone, E. A. (2008). A developmental study of risky decisions on the cake gambling task: Age and gender analyses of probability estimation and reward evaluation. Developmental neuropsychology, 33(2), 179-196. doi: 10.1080/87565640701884287.

Verbruggen, F. \& Logan, G. (2008). Response inhibition in the stop-signal paradigm. Trends Cognitive Science, 12(11), 418-424. doi: 10.1016/j.tics.2008.07.005.

Welsh, M., Pennington, B. \& Groisser, D. (1991). A normative developmental study of executive function: a window on prefrontal function in children. Developmental neuropsychology, 7(2), 131-149. doi: 10.1080/87565649109540483.

Wright, L., Lipszyc, J., Dupuis, A., Thayapararajah, S. W. \& Schachar, R. (2014). Response inhibition and psychopathology: A metaanalysis of go/no-go task performance. Journal of Abnormal Psychology, 123(2), 429. doi: $10.1037 / \mathrm{a} 0036295$

Zelazo, P., Muller, U., Frye, D. \& Marcovitch, S. (2003). The development of executive function in early childhood. Monographs of the Society for Research in Child Development, 68(3), 137. doi: 10.1111/j.0037-976X.2003.00260.x. 